\title{
Revisit of statistical selection algorithm for peer-to-peer system
}

\begin{abstract}
Over the years, the distributed computing environment has been developed so fast that thereô a need to develop an effective selection algorithm for it. Loo [9] has proposed a statistical selection algorithm with the same objective and run in the multicast / broadcast environment that has been proved that it is the best among others algorithm in the same platform in terms of the number of messages and the number of rounds needed to complete the searching process. This work revisited the work by Loo [9] through simulation. The result obtained from the simulation is almost the same with [9] in terms of the number of messages needed and the number of rounds needed.
\end{abstract}

Keyword: Peer-to-peer system; Statistical selection algorithm 